MITSUBISHI ELECTRIC RESEARCH LABORATORIES

http://www.merl.com

\title{
Modeling and Forecasting Self-Similar Power Load Due to EV Fast Chargers
}

\author{
Korolko, N.; Sahinoglu, Z.; Nikovski, D.N. \\ TR2015-105 August 2015
}

\begin{abstract}
In this article, we consider modeling and prediction of power loads due to fast charging stations for plug-in electric vehicles. The first part of the project is to simulate work of a fast charger activity by exploiting empirical data that characterize EV user behavior. The second part describes the time series obtained by this simulator and its properties. We show that the power load aggregated over a number of fast chargers (after deseasonalizing and elimination of the linear trend) is a self-similar process with the Hurst parameter $0.57 ; \mathrm{H} ; 0.67$, where $\mathrm{H}$ varies depending on the multiplexing level. The main contribution of the paper is empirical evidence that a fitted fractional autoregressive integrated moving average (fARIMA) model taking into account self-similarity of the load time series can yield high quality short-term forecasts when $\mathrm{H}$ is large enough. Namely, the fitted fARIMA model uniformly outperforms regular ARIMA algorithms in terms of root-mean-square error for predictions with time horizon up to 120 minutes for $\mathrm{H}$ greater than or equal to 0.639. Moreover, we show that the fARIMA advantage on average grows as a function of the Hurst exponent $\mathrm{H}$. Computational experiments demonstrate that this edge is stably greater than $1.1 \%$ and can be as high as $5-7 \%$ for some scenarios.
\end{abstract}

2015 IEEE Transactions on Smart Grid

This work may not be copied or reproduced in whole or in part for any commercial purpose. Permission to copy in whole or in part without payment of fee is granted for nonprofit educational and research purposes provided that all such whole or partial copies include the following: a notice that such copying is by permission of Mitsubishi Electric Research Laboratories, Inc.; an acknowledgment of the authors and individual contributions to the work; and all applicable portions of the copyright notice. Copying, reproduction, or republishing for any other purpose shall require a license with payment of fee to Mitsubishi Electric Research Laboratories, Inc. All rights reserved.

Copyright (c) Mitsubishi Electric Research Laboratories, Inc., 2015

201 Broadway, Cambridge, Massachusetts 02139 



\title{
Modeling and Forecasting Self-Similar Power Load Due to EV Fast Chargers
}

\author{
Nikita Korolko, Zafer Sahinoglu, Senior Member, IEEE, and Daniel Nikovski, Member, IEEE
}

\begin{abstract}
In this article, we consider modeling and prediction of power loads due to fast charging stations for plug-in electric vehicles. The first part of the project is to simulate work of a fast charger activity by exploiting empirical data that characterize EV user behavior. The second part describes the time series obtained by this simulator and its properties. We show that the power load aggregated over a number of fast chargers (after deseasonalizing and elimination of the linear trend) is a self-similar process with the Hurst parameter $0.57<H<0.67$, where $H$ varies depending on the multiplexing level. The main contribution of the paper is empirical evidence that a fitted fractional autoregressive integrated moving average (fARIMA) model taking into account self-similarity of the load time series can yield high quality short-term forecasts when $H$ is large enough. Namely, the fitted fARIMA model uniformly outperforms regular ARIMA algorithms in terms of root-mean-square error for predictions with time horizon up to $\mathbf{1 2 0}$ minutes for $H \geq 0.639$. Moreover, we show that the fARIMA advantage on average grows as a function of the Hurst exponent $H$. Computational experiments demonstrate that this edge is stably greater than $1.1 \%$ and can be as high as $5-7 \%$ for some scenarios.
\end{abstract}

Index Terms-Demand forecasting, load modeling, selfsimilarity.

\section{INTRODUCTION}

$\mathbf{W}$ ITH the growing concern over green house effect and climate changes, transportation systems are changing rapidly with the increasing adoption of electric vehicles (EV) and plug-in hybrid electric vehicles (PHEV). The global emissions reduction objectives (e.g. $20 \% \mathrm{CO}_{2}$ reduction by 2020 in USA, Europe and Japan [1]) strongly suggest higher penetration level of EVs, renewable energy sources and demand response applications in the future. On the other hand, there are some barriers associated with the large-scale adoption of EVs, including long charging duration, range anxiety and cost of EVs. Introduction of fast charger stations (CHAdeMO $50 \mathrm{~kW}$, SAE $60 \mathrm{~kW}$, Tesla $120 \mathrm{~kW}$ ) in comparison with standard chargers (Level 1 - 2-3.3 kW, Level 2 - 4-7.2 kW) shorten charging durations to tens of minutes. However, this produces an even bigger challenge for electric utilities. The main reason is that such a high level of fast charger energy consumption makes the aggregate load more irregular and difficult to predict. In order to adequately respond to the emerging challenges in systems planning and operations, it is necessary to know how to simulate and forecast load demand due to a large number of EV fast chargers. We address these two issues in this work.

There are several studies that discuss different statistical approaches applied to modeling EV driving schedules and impact on a distribution system due to EV charging [2] - [6]. In
[2] charging load is simulated in various scenarios taking into consideration location of charging (domestic/public) and ability to control the time of charge (uncontrolled/off-peak/smart). The probabilistic distributions of initial state of charge, daily distance driven, etc are derived in [2] from UK National Statistics that includes information about all types vehicles rather than EVs only. Lee et al. introduce in [3] a combination of Bayesian temporal distribution models and Markov-chain technique to synthesize individual driving cycles. The modeling of PHEV load using National Travel Survey (Finland) and considering different cases with respect to a season of the year and a day of the week is presented in [4]. Reference [5] models a power load as a linear combination of the convenience driven mode and the cost driven mode. The first approach assumes the charging load follows the same shape as the travel pattern curve while the second one presumes that the load follows a "valley-filling" pattern. Another prominent study concentrated on simultaions of usage pattern of a fast charging station (rather than standard charger) is published by National Renewable Energy Laboratory (NREL) [6]. The grid impact and opportunity for integration of renewables is also discussed in [6].

Despite the fact that the majority of modeling techniques introduced in [2] - [6] already incorporate real-world travel data, the reliability and relevance of surveys collected over all types of vehicles and standard charging stations can be an issue for generation of EVs load demand due to fast chargers. In our study, we exploit more applicable and recent data from Idaho National Laboratory (INL, Q3 2013) [7] specializing on EV fast chargers only. Moreover, in the forecasting part of the paper we propose a novel approach based on self-similarity of deseasonalized load.

This paper is organized as follows. Section 2 describes EV driving schedule data as well as temporal fast charging distributions obtained from NREL, Alternative Fuel Data Center and INL. The primary result of this section is an algorithm that models the work of a fast charger given an EV population that this charger serves. The output of the algorithm is a time series which is equal to the immediate aggregate load due to EV fast charging after multiplexing over a large number of stations. The third section includes the description of load characteristics and the empirical proof that the obtained stochastic process after some invertible transformations (deseasonalizing and elimination of the linear trend) is self-similar. Moreover, several different Hurst parameter calculation methods are discussed and corresponding estimates of $H$ are obtained. The self-similarity of the time series makes fractional autoregressive integrated moving average (fARIMA) 
model with non-integer differencing parameter a reasonable candidate for forecasting the future values of the load demand. Forecasting algorithms that we use are discussed in section 4. In the last section, we provide simulation results and compare the performance of standard ARIMA models with fitted fARIMA models.

The main contribution of the paper is a proof of selfsimilarity of the deseasonalized aggregate EV fast charging load which justifies implementation of a fARIMA forecasting algorithm. This augmented fractional autoregressive integrated moving average model outperforms the best fitted ARIMA model for relatively short-term forecasts with time horizons up to 120 minutes when $H$ is large enough.

\section{Modeling EV Charging Behaviors}

In this section, we develop an algorithm that simulates the usage pattern of a single fast charger station by a set of EVs, based on the real-world data given in [6] - [11]. Our algorithm can be considered as an extension of a model introduced by Simpson and Markel [6] since their model exploits universal information and assumptions about EV charging behavior. The generic assumptions we inherit from Simpson and Markel [6], as well as additional key augmentations are discussed in the next subsection.

\section{A. Assumptions}

1) The number of charging stations is $M$, each with one charging port (by default, $M=50$ ). This parameter represents an aggregation level of load demand explicitly defined below in equation 1 .

2) The fleet size, that is local population of EVs that can charge at a station, is denoted by $N$. According to [10], there are 286,000 EVs sold in the US since 2010 and 2,346 fast charging outlets [11], what yields a national average fleet size $\bar{N}=122$. In our study, we set a default value $N=100$.

3) EV owners will charge most frequently at home. This conjecture is supported by the Electric Power Research Institute charging pyramid [8]. There are only two reasons why an EV owner will not plug a vehicle in at home on any given day. Either the operator forgets to do it with some probability (so-called forgetfulness factor, denoted as $\gamma$ ) or a trip that started the day before is not finished by $12 \mathrm{am}$. The latter scenario implies that the driver is absent at home during the night. In our study, the forgetfulness factor $\gamma$ is set to $10 \%$ by default.

4) EV energy consumption is $300 \mathrm{Wh} / \mathrm{mi}$ while on the move.

5) EVs start fast charging when their state of charge (SoC) is within $10-40 \%$ and terminate charging when $\mathrm{SoC}$ reaches $70-100 \%$. Initial SoC is uniformly distributed between $10-90 \%$.

6) Efficiency of fast chargers is $85 \%$ due to anticipated losses from internal battery resistance and AC-to-DC power conversion.

7) Fast charger power level is $50 \mathrm{~kW}$ with $25 \%$ safety factor (total $62.5 \mathrm{~kW}$ required).
Table I

TYPES OF THE EVS AND THEIR PARAMETERS

\begin{tabular}{|l|c|c|}
\hline EV type & Capacity C, $\mathrm{kWh}$ & Market share P(EV), \% \\
\hline Chevrolet Volt & 16.5 & 44.28 \\
\hline Nissan LEAF & 24 & 35.71 \\
\hline Tesla Model S & 65 & 20.01 \\
\hline
\end{tabular}

In addition to the assumptions from [6] listed above, we introduce new extensions to the model that we build as follows:

8) There are $n$ different types of EVs denoted as $E V_{1}, \ldots, E V_{n}$. The battery capacity and the percentage of EV type $r$ in the population is denoted $C_{r}$ and $P\left(E V_{r}\right)$, respectively. To be more precise, according to the Alternative Fuels Data Center [9] and available EV and PHEV sales statistics in the US in 201014 there are three predominant models that constitute the majority of the plug-in EV market: Chevrolet Volt (30.5\%), Nissan LEAF (24.8\%) and Tesla S (13.2\%). After elimination of hybrid EVs and models with a small population we consider a scenario with only $3 \mathrm{EV}$ types. Their characteristics (capacity and market share after normalization) are represented in the Table I.

9) In order to generate the charging load, we consider a horizon of $D$ (by default $D=25$ ) consecutive and identical days. More precisely, following [6] we assume that all days are indistinguishable from the perspective of the distributions listed below:

a) EV departure time when EV trip starts with empirical probability density function $f_{d t}(t), t \in[0 ; 24]$ hours;

b) EV average speed with probability density $f_{s}(x), x \in$ [0;90] miles/hour;

c) EV trip length with probability density $f_{l}(x), x \in$ $[0 ; 50]$ miles.

These distributions are explicitly represented in [6] and based on the information about 149,000 individual trips gathered by Puget Sound Regional Council in 2008 in Seattle metropolitan area.

10) Another empirical data that we want to incorporate into the load modeling is fast charging demand versus the time of day and duration of fast charging. Empirical density functions $f_{a r}(t), t \in[0 ; 24]$ hours, for arrival time to the fast charger and $f_{c t}(t), t \in[0 ; 60]$ minutes, for charging duration are obtained from "The EV Project" by INL [7].

11) The probability $\delta$ that an EV will commute during any given day is $5 / 7$. This assumption represents a scenario when an operator uses her EV on average 5 days per week.

Having introduced assumptions A1-A11, now it is possible to simulate the activity of each of the $M$ fast chargers during $D$ consecutive days. This work is described by a continuous stochastic process $W^{i}(t)$ representing an immediate load demand at the fast charger $i$ :

$W^{i}(t)=62.5 \cdot \mathbb{I}($ The station $i$ is $\mathrm{ON}$ at time $\mathrm{t})$, 


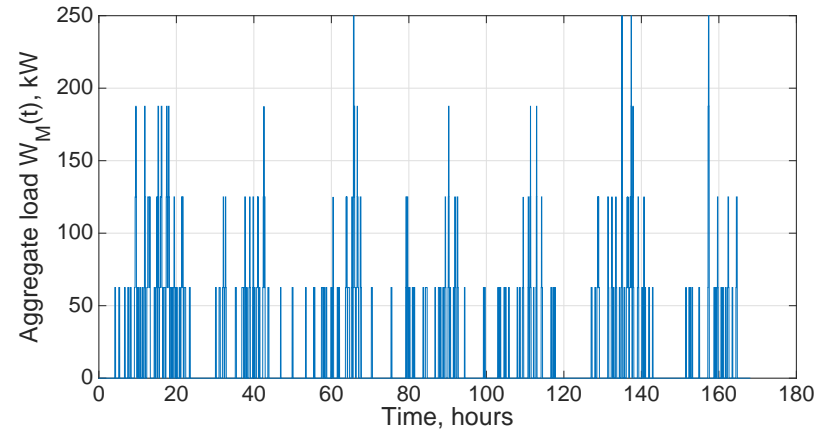

Figure 1. Aggregate immediate load $W_{M}(t)$ for a horizon $D=7$ days.

where coefficient $62.5=50 \times 1.25$ represents $50 \mathrm{~kW}$ load with $25 \%$ safety factor and $\mathbb{I}$ is an indicator function. In this case the immediate total load demand aggregated over $M$ fast chargers is defined as follows:

$$
W_{M}(t)=\sum_{i=1}^{M} W^{i}(t)
$$

We discretize $W_{M}(t)$ at a sampling frequency of 50 observations per hour. In the later sections we continue to use notation $t$ as a sample index for discrete signals rather than a continuous time parameter.

\section{B. Generation of the aggregate charging schedule $W_{M}(t)$}

In this subsection, we describe the algorithm for modeling the time series $W_{M}(t)$ that represents the power load aggregated over $M$ fast chargers.

1) Repeat steps 2-18 for each of $d=1 \ldots D$ days.

2) Repeat steps 3-12 for each of EV $j=1 \ldots N$ in the fleet.

3) For fixed $j$, define a type $k \in\{1 \ldots n\}$ of $j$-th EV by finding $k$ s.t.

$$
1+\sum_{r=1}^{k-1}\left[P\left(E V_{r}\right) \cdot N\right] \leq j \leq \sum_{r=1}^{k}\left[P\left(E V_{r}\right) \cdot N\right]
$$

where $n=3$ for our study and market shares $P\left(E V_{r}\right)$ are described in assumption A8.

4) If $d=1$, then generate an initial $S o C_{j} \in \mathbb{U}[0.1 ; 0.9]$ according to A5.

5) Generate $\delta \in \mathbb{B}(5 / 7)$ as defined in A11. If $\delta=0$, then $j$ th EV does not commute on that day and algorithm goes to step 17 with binary fast charging indicator $F C_{j}=0$; otherwise, it goes to step 6.

6) Generate departure time $d t_{j}$, average speed $s_{j}$ and length of the trip $l_{j}$ according to corresponding distributions from A9.

7) Check if the fast charging is necessary by examining inequality

$$
0.3 \cdot l_{j} \geq\left(S o C_{j}-0.1\right) C_{k(j)},
$$

where $0.3 \cdot l_{j}$ is equal to amount of energy required for a trip of length $l_{j}$ (A4), and RHS equals current battery charge reduced by $10 \%$ (A5). If this inequality holds, then set binary fast charging indicator $F C_{j}=1$ and go to step 8; otherwise, set $F C_{j}=0$, update

$$
S o C_{j}=S o C_{j}-\frac{0.3 l_{j}}{C_{k(j)}}
$$

according to A4 and go to step 17.

8) Calculate $t_{j}(0.4)$ and $t_{j}(0.1)$ when SoC of $j$-th EV will be $40 \%$ and $10 \%$ (A5), respectively, using average speed $s_{j}$ and energy consumption rate $0.3 \mathrm{kWh} / \mathrm{mi}(\mathrm{A} 4)$ :

$$
t_{j}(\xi)=d t_{j}+\frac{\left(S o C_{j}-\xi\right) C_{k(j)}}{0.3 s_{j}}
$$

where $\xi \in\{0.1 ; 0.4\}$.

9) Generate a moment of arrival $a r_{j}$ to a fast charging station of $j$-th EV inside time interval $\left[t_{j}(0.4) ; t_{j}(0.1)\right]$ according to normalized distribution

$$
\bar{f}_{a r}(t)=\frac{f_{a r}(t)}{\int_{t_{j}(0.4)}^{t_{j}(0.1)} f_{a r}(u) d u}, \quad t \in\left[t_{j}(0.4) ; t_{j}(0.1)\right],
$$

derived from density $f_{a r}(t)$ that described in A10. Update

$$
S o C_{j}=S o C_{j}-\frac{0.3 s_{j}\left(a r_{j}-d t_{j}\right)}{C_{k(j)}} .
$$

10) Calculate $t_{j}(0.7)$ and $t_{j}(1)$ when SoC of $j$-th EV will reach $70 \%$ and $100 \%$ level (A5), respectively, given $85 \%$ efficiency of charger (A6) and its power level $50 \mathrm{~kW}$ (A7):

$$
t_{j}(\eta)=a r_{j}+\frac{\left(\eta-S o C_{j}\right) C_{k(j)}}{0.85 \cdot 50},
$$

where $\eta \in\{0.7 ; 1\}$.

11) Generate charging time $c t_{j}$ inside time interval $\left[t_{j}(0.7)-a r_{j} ; t_{j}(1)-a r_{j}\right]$ according to normalized distribution $\bar{f}_{c t}(t), t \in\left[t_{j}(0.7)-a r_{j} ; t_{j}(1)-a r_{j}\right]$ derived from $f_{c t}(t)$ that described in A10 similarly to step 9.

12) Update SoC after charging and finishing the trip of length $l_{j}$ according to formula

$S o C_{j}=S o C_{j}+\frac{c t_{j} \cdot 0.85 \cdot 50}{C_{k(j)}}-\frac{0.3\left(l_{j}-s_{j}\left(a r_{j}-d t_{j}\right)\right)}{C_{k(j)}}$

13) At this point, we obtain $V=\sum_{j=1}^{N} F C_{j}$ intervals of the form $\left[a r_{j}, a r_{j}+c t_{j}\right]$ for some $j \in\{1 \ldots N\}$ that correspond to charging events. If some intervals intersect, for instance the first $k$ intervals:

$$
\begin{array}{r}
{\left[a r_{1}, a r_{1}+c t_{1}\right], \ldots,\left[a r_{k}, a r_{k}+c t_{k}\right]} \\
\text { s.t. } a r_{1}<a r_{2}<\cdots<a r_{k}
\end{array}
$$

then we organize a queue of $k$ users with $c t_{1}, \ldots, c t_{k}$ service times according to first come first served rule. It is also necessary to assume 1.2 minute interval (1/50 h) between users in a queue because the next EV driver cannot immediately start charging when the current one stops doing it. In other words, we shift intersecting 
intervals ordering them one by one, saving their length and making them disjoint.

14) Based on rearrangements defined in step 13, construct indicator function $W(t)$, which is equal to 62.5 if at time $t$ the fast charging station is on (A7).

15) Repeat steps 16-18 for each of EV $j=1 \ldots N$ in the fleet.

16) Given rearrangements from step 13 , calculate the waiting time of $j$-th $\mathrm{EV}$ in a queue $w t_{j}$ for fast charging.

17) Calculate the time of arrival of $j$-th EV to home:

$$
h_{j}=d t_{j}+\delta \cdot \frac{l_{j}}{s_{j}}+F C_{j} \cdot\left(c t_{j}+w t_{j}\right) .
$$

18) If time $h_{j}$ is after midnight, then $\mathrm{EV}$ is not charged at home according to $\mathrm{A} 3$. Otherwise, generate $\gamma \in \mathbb{B}(0.9)$. If $\gamma=1$, then SoC of $j$-th EV is updated as follows

$$
S o C_{j}=\max \left(S o C_{j}, 0.8\right),
$$

what represents a home charging up to $80 \%$. If $\gamma=0$, then EV user forgets to charge her vehicle at home and $S o C_{j}$ remains unchanged (A3).

19) Repeat steps 1-18 of the generating algorithm for each of the $M=50$ stations. Obtain the set of time series $W^{i}(t), i=1 \ldots M$ for $t=1, \ldots, 1200 D$, since $D$ days $\cdot 24$ hours $\cdot 50 \frac{\text { observations }}{\text { hour }}=1200 D$ observations.

20) Aggregate immediate loads $W^{i}(t)$ with respect to $i=$ $1 \ldots M$ to obtain the total load $W_{M}(t)$ according to (1).

A part of the resulting time series $W_{M}(t)$ for $M=50$ stations and a horizon of $D=7$ days (out of total 25) is represented in Fig. 1.

\section{LOAD CHARACTERISTICS}

One of the main objectives for electric utilities is to build an accurate forecasting algorithm for future values of the time series $W_{M}(t)$ obtained in section 2. In order to make this predictor more accurate, we discuss in this section several invertible transformations and useful properties of the load $W_{M}(t)$ that facilitate reduction of the forecast error.

\section{A. Seasonal Decomposition}

First of all, the equivalence of $D$ days from the perspective of probabilistic distributions describing EV users behavior (departure time, average speed and distance, arrival times to the fast charger) implies the presence of strong periodicity. In other words, one may expect that the number of charging EVs at any given time doesn't differ statistically across subsequent days. This conjecture can be also supported by observation of Fig. 1 with 7 distinct cycles of similar length each of which corresponds to one day.

A simple way to examine serial dependencies is to plot autocorrelation function of the aggregate load $W_{M}(t)$ [12]. The presence of local maxima at lags which are multiples of 1200 (what corresponds to $1200 / 50=24$ hours) implies periodicity of the initial time series with the period of one day.

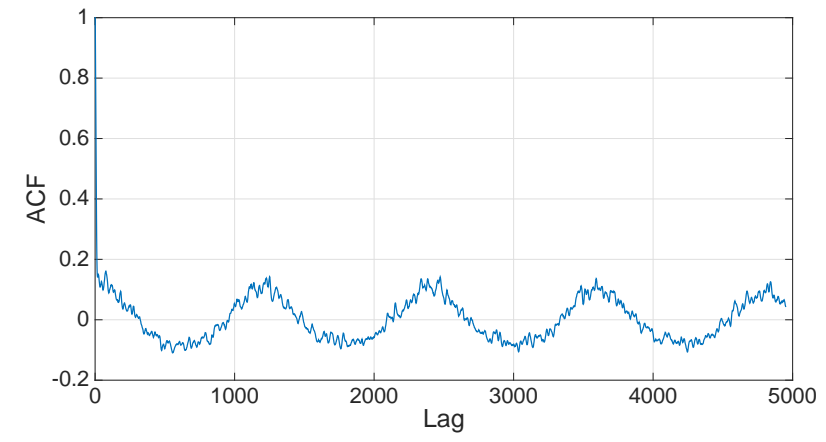

Figure 2. Autocorrelation function of load $W_{M}(t)$.

Therefore, it is necessary to decompose the aggregate load $W_{M}(t)$ for $t=1 \ldots 1200 D$ into three separate components:

$$
W_{M}(t)=L T(t)+S(t)+Y(t),
$$

where $L T(t)$ represents a linear trend, $S(t)$ a seasonal component and $Y(t)$ the remaining stochastic variability.

Linear trend $L T(t)=a \cdot t+b$ for $t=1 \ldots 1200 D$ is uniquely defined by two unknown parameters $a$ and $b$ which can be easily obtained via linear regression.

After estimating and removing the linear trend $L T(t)$, the seasonal component $S(t)$ for $t=1 \ldots 1200 D$ that consists of $D$ identical cycles of length 1200 observations has to be computed. There are many different ways to estimate the seasonal component. It is important to choose the one that gives the best fit to the time series being considered. In our study, we consider 4 algorithms for extraction of $S(t)$. They are thoroughly defined in [12]:

1) Average day technique. Calculation of the arithmetic mean of time series values over $D$ days for all $t=$ $1 \ldots 1200$. The resulting seasonal component is denoted as $S_{m}$.

2) Median day technique. Calculation of median (0.5 quantile) of time series values over $D$ days for all $t=$ $1 \ldots 1200\left(S_{\text {med }}\right)$.

3) Moving average technique. The first step of the method is estimation of the trend by application of the moving average filter in order to eliminate the seasonality and dampen the noise. In our case the length of this filter is 1200 observations. The second step is calculation of the average deviations from the trend. Finally, the coefficients $s_{i}$, for $i=1 \ldots 1200$ of the extracted seasonal component $\left(S_{m a}\right)$ are normalized average deviations from the trend.

4) Spectral decomposition. Fitting a sinusoid shifted in time and space with a period 1200 to the initial time series $\left(S_{\text {sin }}\right)$.

It is worth mentioning that the first $\left(S_{m}\right)$ and the third $\left(S_{m a}\right)$ methods applied to the aggregate load $W_{M}(t)$ yield very similar seasonality components (Fig. 3). On the other hand, in our model which will be thoroughly described in section 5 , the total difference between the corresponding seasonal components can be as high as

$$
\left\|S_{m}-S_{m a}\right\|_{1}=2066.8 \mathrm{~kW} .
$$




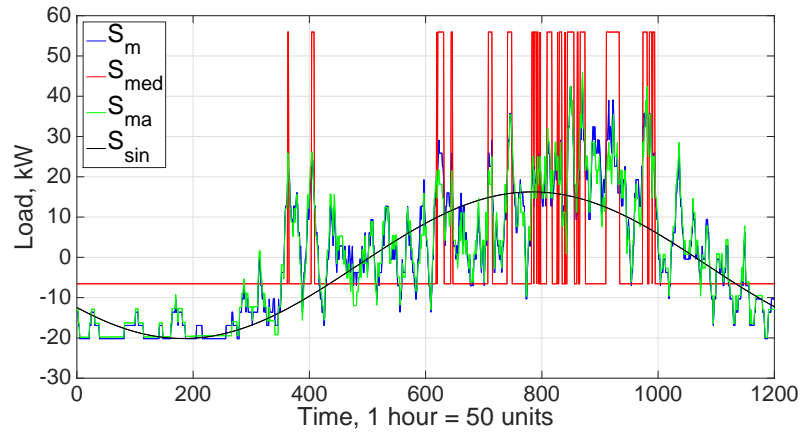

Figure 3. Seasonal components extracted from $W_{M}(t)$ by different methods.

This difference may dramatically impact the prediction error. Therefore, we consider all four methods of the seasonal component computation in order to find the best fit.

The final step of the seasonal decomposition is to extract the deseasonalized residual load $Y(t)$ according to the following formula:

$$
Y(t)=W_{M}(t)-L T(t)-S(t) .
$$

We define the residual deseasonalized loads corresponding to the 4 methods described above as $Y_{m}, Y_{m e d}, Y_{m a}$ and $Y_{\sin }$. Finally, it is necessary to test the stationarity of these stochastic processes $Y_{i}(t), i \in\{m$, med, ma, sin $\}$ by Augmented Dickey-Fuller (ADF) test [13], Kwiatkowski, Phillips, Schmidt, and Shin (KPSS) test [14], etc.

\section{B. Self-Similarity of the Deseasonalized Load}

A key property of the deseasonalized load that improves the accuracy of forecasting algorithm is self-similarity [15]. The aggregate load $W_{M}(t)$ exhibits many bursts at different time scales when multiplexed over $M$ stations (Fig. 4) .

It is important to note that similar behavior with extended periods above/below the mean can be observed for the deseasonalized load $Y(t)$ (Fig. 5). This behavior of the time series is known as "Joseph effect" [16] which is one of the distinctive features of the self-similar processes. The degree of self-similarity of a time series can be measured by the Hurst exponent $0<H<1$ [17]. Let $\{Y(t), t=1,2, \ldots\}$ be a wide-sense stationary time series with both mean and autocorrelation functions time invariant. For each $m \in \mathbb{N}$, denote the $m$-aggregated time series $Y^{(m)}(t), t=1,2, \ldots$ according to the rule:

$$
Y^{(m)}(t)=\frac{1}{m}(Y(t m-m+1)+\cdots+Y(t m)), \quad t \geq 1 .
$$

Then the time series $Y(t)$ is called $H$-self-similar if for all $m \in \mathbb{N}$, the aggregate time series $Y^{(m)}$ has the same distribution as $Y$ rescaled by a constant $m^{H}$. In other words,

$$
Y(t) \stackrel{d}{=} m^{-H} \sum_{i=t m-m+1}^{t m} Y(i) \quad \text { for all } m \in \mathbb{N} .
$$

The Hurst parameter $H$ expresses the speed of decay of the time series autocorrelation function $\operatorname{ACF}(Y)$.

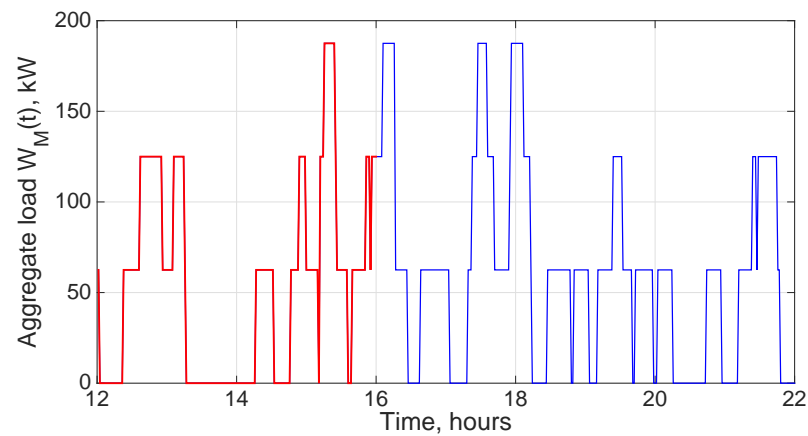

(a) 1 hour $=50$ units

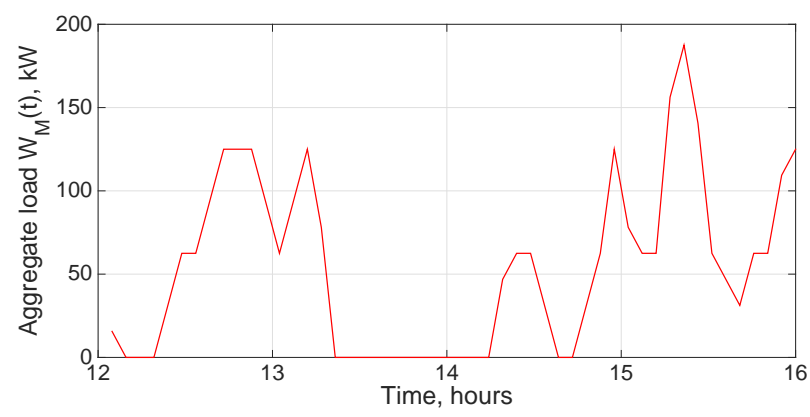

(b) 1 hour $=200$ units

Figure 4. Fragment of load $W_{M}(t)$ at different multiplex levels. Red color indicates the same segment of load.

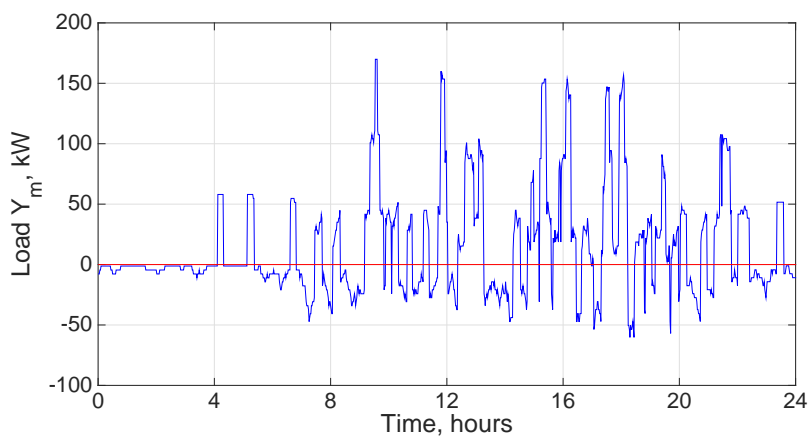

Figure 5. Deseasonalized load $Y_{m}(t)$. Red line corresponds to the mean value.

It is known that every self-similar time series with the Hurst exponent $\frac{1}{2}<H<1$ has an additional property of longrange dependence [18]. Such a time series has autocorrelation coefficients $r(k) \sim k^{-\beta}$ as $k \rightarrow \infty$, where $H=1-\beta / 2$.

There are several statistical methods for testing selfsimilarity and estimation of the Hurst parameter $H$ for a given time series [17]. In this study, we exploit the variance-time plot relying on the slowly decaying variance of a self-similar series, $\mathrm{R} / \mathrm{S}$ analysis using the rescaled range statistic and periodogram method estimating the slope of the power spectrum of the series as frequency approaches zero.

The computational results of these methods confirming the self-similarity of the deseasonalized load $Y(t)$ with the Hurst parameter $H$ in the interval $[0.57 ; 0.67]$ are presented in 
section 5 .

\section{ForecAsting Algorithms}

In a highly competitive energy market load forecasting is an essential problem to electric utilities for many reasons [12]. The electricity price variability leads to the significant risks for energy suppliers that have to manage contracts of different types and durations. Short-term forecasts are very important to ensure reliability of electricity supply to consumers, while long-term load demand predictors are used as the basis for future development of the utilities. Thus, load forecasting has become the central process in the planning and operation of energy suppliers with huge monetary penalties for prediction errors. This is why increasing forecast quality even for small percentage may lead to significant economic value for utilities.

The seasonal decomposition (2) described in subsection 3(A) implies that the load $W_{M}(t)$ consists of two deterministic terms (linear trend $L T(t)$ and a seasonal component $S(t)$ ) that can be evaluated based on the historical data. Hence, both processes $L T(t)$ and $S(t)$ can be used for prediction of the future values of the total load $W_{M}(t)$. Therefore, the only stochasticity that requires accurate characterization is contained in the process $Y(t)$. Below we discuss three different methods for forecasting the deseasonalized load $Y(t)$.

1) Baseline method.

The simplest predictor for the process $Y(t)$ is the mean value $\mathbb{E} Y$ estimated based on the observations from the training set. In this case, the predictor for the aggregate load $W_{M}(t)$ is just the historical average of demand for the same time of the day.

2) Autoregressive integrated moving average model $\operatorname{ARIMA}(p, d, q)$.

Non-negative integer parameters $p, d$ and $q$ correspond to the order of autoregressive (AR), integrated (I) and moving average (MA) parts of the method, respectively [19]. The principal idea of the model is to build a forecast for the future values of the time series $Y(t)$ as a linear function of previous values $Y(t-1), Y(t-2), \ldots$ and the error terms $\varepsilon(t), \varepsilon(t-1), \ldots$ which are assumed to be i.i.d. random variables with zero mean. In terms of the lag operator $B^{i} Y(t)=Y(t-i)$ the ARIMA model can be represented by the following equation:

$$
\begin{array}{rl}
\left(1-\sum_{i=1}^{p} \alpha_{i} B^{i}\right)(1-B)^{d} & Y(t) \\
& =\left(1+\sum_{j=1}^{q} \beta_{j} B^{j}\right) \varepsilon(t),
\end{array}
$$

where $\alpha_{i}, i=1 \ldots p$ and $\beta_{j}, j=1 \ldots q$ are parameters of the AR and MA parts, respectively. Given a time series $\{Y(t), t=1,2 \ldots\}$ the problem of fitting the best ARIMA algorithm into it consists of two parts. The first one is the model selection problem, that is choosing the order parameters $p, d, q$, for instance, based on one of the information criteria (IC): Bayesian (BIC), Akaike (AIC), etc. The second part is the estimation of unknown coefficients $\alpha_{i}, i=1 \ldots p$ and $\beta_{j}, j=1 \ldots q$ of the corresponding AR and MA parts using auxiliary maximum likelihood or least squares optimization problem. Both these procedures can be executed automatically by different software including $\mathrm{R}$ package 'forecast' used in this study [20].

3) Fractional autoregressive integrated moving average model fARIMA $(p, d, q)$.

This method is a generalization of the previous one allowing integrated parameter $d$ to take non-integer values, which was designed in order to capture both short-range and long-range dependence of the timeseries simultaneously [21]. The fractional differencing operator in this case is defined as follows

$$
(1-B)^{d}=\sum_{i=0}^{\infty}\left(\begin{array}{l}
d \\
i
\end{array}\right)(-B)^{i},
$$

where $\left(\begin{array}{c}d \\ i\end{array}\right)=\Gamma(d+1) /[\Gamma(k+1) \Gamma(d-k+1)]$ and $\Gamma$ denotes the Gamma function.

If parameter $d$ in the definition (3) is equal to 0 , then fARIMA $(p, d, q)$ model is the usual short-range dependent $\operatorname{ARMA}(p, q)$ model. On the other hand, it is known that fARIMA(p,d,q) process with $d \in(0,0.5)$ exhibits persistence, where parameter $d$ indicates the strength of long-range dependence similar to the Hurst parameter of self-similar processes [22]. In fact, the relation between these two parameters is $H=d+0.5$.

Similar to the ARIMA case, it is possible to fit the fARIMA(p,d,q) model to the time series $Y(t)$ using the function 'arfima' from $\mathrm{R}$ package 'forecast'.

\section{Computational Results}

In this section, we implement all modeling algorithms and compare the performance of the forecasting methods described in the previous sections. First of all, following the procedures illustrated in section 2 , it is necessary to simulate the immediate aggregate load $W_{M}(t)$ (part of which is represented in Fig. 1) for $D=25$ consecutive days, $M=50$ fast chargers with 1 charging slot and a local fleet consisting of $N=100 \mathrm{EVs}$ for each of the stations. After this, the total set of $25 \cdot 1200=30000$ observations is split into a training set of 24000 successive observations that correspond to the horizon of the first 20 days and a testing set of the remaining 6000 observations (5 days).

The next step is seasonal decomposition of the load demand $W_{M}(t)$ into three sub-processes according to formula (2). Coefficients of the linear trend $L T(t)=a \cdot t+b$ are estimated on the training set with MATLAB function 'polyfit'. The coefficient values

$$
a=-0.0000187, \quad b=21.678,
$$

imply that the best linear predictor for the $W_{M}(t)$ is a constant function (since the slope is negligibly small), which is equal to the mean load demand $21.678 \mathrm{~kW}$. Having computed 4 seasonal components $S_{m}, S_{m e d}, S_{m a}$ and $S_{s i n}$ as stated in subsection 3(A), one can obtain the corresponding deseasonalized loads $Y_{m}, Y_{m e d}, Y_{m a}$ and $Y_{s i n}$. For these 4 extracted processes it is necessary to run ADF and KPSS stationarity 
Table II

RESULTS OF STATISTICAL STATIONARITY TESTS

\begin{tabular}{|l|c|c|}
\hline Deseasonalized Load & ADF value & KPSS value \\
\hline$Y_{m}$ & -31.2647 & 2.4197 \\
\hline$Y_{m e d}$ & -36.8286 & 1.7646 \\
\hline$Y_{m a}$ & -31.2696 & 2.4110 \\
\hline$Y_{\text {sin }}$ & -31.0270 & 2.2500 \\
\hline \hline Critical Value & -1.9416 & 0.1460 \\
\hline
\end{tabular}

Table III

ESTIMATION OF THE HURST PARAMETERS

\begin{tabular}{|l|c|c|c|c|}
\hline Load & Time-variance plot & RS analysis & Periodogram & Average \\
\hline$Y_{m}$ & 0.6389 & 0.6245 & 0.6298 & $\mathbf{0 . 6 3 1 0}$ \\
\hline$Y_{m e d}$ & 0.6343 & 0.6304 & 0.6306 & $\mathbf{0 . 6 3 1 7}$ \\
\hline$Y_{m a}$ & 0.6363 & 0.6284 & 0.6332 & $\mathbf{0 . 6 3 2 6}$ \\
\hline$Y_{\text {sin }}$ & 0.6153 & 0.6071 & 0.6093 & $\mathbf{0 . 6 1 0 5}$ \\
\hline
\end{tabular}

tests, for instance, taking advantage of MATLAB functions 'adftest' and 'kpsstest' from the Econometrics toolbox. The output statistical values calculated on the training set are listed in the Table II. Since for the ADF test all residuals $Y_{i}$ for $i \in\{m$, med, $m a, \sin \}$ yield test statistics below a threshold -1.9416, we accept the hypothesis that all four deseasonalized loads $Y_{i}$ are stationary. Similarly, for the second test KPSS statistic values above the critical value 0.1460 imply stationarity of stochastic processes $Y_{i}$ for $i \in\{m$, med, ma, sin $\}$.

The self-similarity of the time series $Y(t)$ is estimated on the training set by three different methods indicated in subsection 3(B) (time-variance plot, R/S analysis, periodogram) and the average values of estimated Hurst exponents are listed in the last column of Table III. For all deseasonalized loads that we consider the Hurst parameter $H$ is in the interval [0.57;0.67], that is $\frac{1}{2}<H<1$. This makes the fARIMA(p,d,q) model with parameter $d=H-0.5$ a reasonable candidate for forecasting. The next part of the computational experiment is a model selection for which only observations from the training set are used. We fit the best $\operatorname{ARIMA}(\mathrm{p}, \mathrm{d}, \mathrm{q})$ model to each of the variants of the time series $Y(t)$ using Bayesian Information Criterion implemented in $\mathrm{R}$ package 'forecast'. After the optimal values of order parameters $p, d, q$ and model coefficients $\alpha_{i}, i=1 \ldots p$, $\beta_{j}, j=1 \ldots q$ are obtained, it is necessary to test the prediction quality of fitted $\operatorname{ARIMA}(\mathrm{p}, \mathrm{d}, \mathrm{q})$ models for time series $Y(t)$ on the testing set. Moreover, in our study we examine different prediction horizons $\tau=1 \ldots 100$. These horizons are equivalent to time units from $1.2 \mathrm{~min}$ to 120 min since the time resolution is 50 observations per hour. The quality measure of a forecasting algorithm $\mathrm{A}$ is the rootmean-square error (RMSE) calculated on the testing set not for stochastic component $Y(t)$ but rather for the initial load $W_{M}(t)$ (2). The RMSE as a function of prediction horizon $\tau=1 \ldots 100$ and algorithm $\mathrm{A}$ is defined as follows

$\operatorname{RMSE}(\tau ; A)=\sqrt{\frac{\sum_{i=1}^{n(\tau)} \sum_{t=1}^{\tau}\left(W_{M}(i+t-1)-\hat{W}_{M}(i+t-1)\right)^{2}}{n(\tau)}}$,

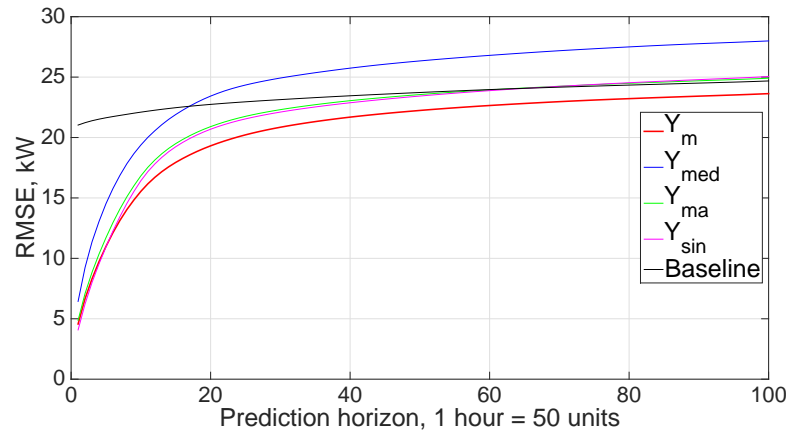

Figure 6. RMSE of the fitted ARIMA models.

where $W_{M}(i)$ is an actual value of the time series, $\hat{W}_{M}(i)=$ $L T(i)+S(i)+\hat{Y}(i)$ is a predicted value according to algorithm $A$ and $n(\tau)=6001-\tau$ is a number of observations in the testing set for a fixed $\tau$.

Five plots on the Fig. 6 represent the forecasting quality of the best fitted ARIMA models to deseasonalized loads $Y_{m}, Y_{m e d}, Y_{m a}$ and $Y_{\text {sin }}$ and a baseline method versus prediction horizon $\tau=1 \ldots 100$. The method corresponding to mean day technique $Y_{m}$ has the smallest RMSE over all time horizons (depicted in red color). The best fitted ARIMA model to the process $Y_{m}$ according to BIC has the following parameters: order $p=3, d=0, q=5$; noise variance $\sigma=13.501$.

The final step of the forecasting model selection problem is to find the fitted fARIMA model to the most accurate deseasonalized load $Y_{m}$ and test its quality in terms of RMSE. The fitted fARIMA with respect to BIC is found by $\mathrm{R}$ package 'forecast' and specified by order parameters $p=1, d=$ $0.1367, q=1$; coefficients $A R(1)=0.8692, M A(1)=$ 0.0511 and noise variance $\sigma=13.599$.

The computational results illustrated on Fig. 7 imply that the fitted fARIMA $(1,0.1367,1)$ model outperforms the best fitted $\operatorname{ARIMA}(3,0,5)$ model for all time horizons $\tau=1, \ldots, 100$ what corresponds to the short-term load forecasts up to 2 hours and the edge can be as high as

$$
\left(\frac{17.68}{16.756}-1\right) \cdot 100 \%=5.61 \%
$$

for $\tau=14$. It is worth mentioning that the differencing order $d=0.1367$ is in almost perfect accordance with estimation of the Hurst exponent $H=0.6310$ for the time series $Y_{m}$, since the theoretical link between these two parameters is $H=$ $d+0.5$.

The following computational experiments consider variations of three most important parameters of the problem: load aggregation level $M$, initial local fleet size $N$ and forgetfulness factor $\gamma$. For each of the scenarios presented in Table IV, we compute the corresponding Hurst exponent and inspect the quality and advantage of the fitted fARIMA model over the the fitted ARIMA algorithm. In order to this, we introduce a function

$$
\operatorname{Edge}(\tau)=\frac{R M S E(\tau ; A R I M A)}{R M S E(\tau ; f A R I M A)}-1
$$




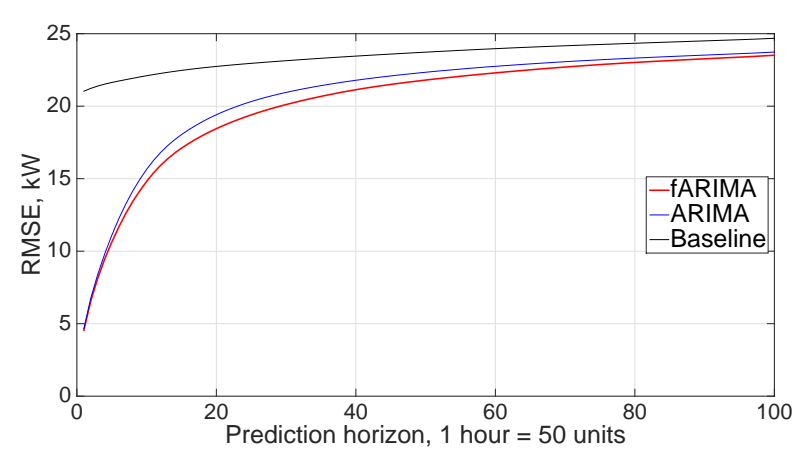

Figure 7. RMSE of the fitted fARIMA and ARIMA models.

for prediction horizons $\tau=1 \ldots 100$. Based on function (4), the quality of the fitted fARIMA algorithm can be measured in terms of the following characteristics:

$$
\begin{aligned}
Q_{\text {fract }} & =\frac{1}{100} \sum_{t=1}^{100} \mathbb{I}\{\operatorname{Edge}(t)>0\}, \\
Q_{\text {mean }} & =\frac{1}{100} \sum_{t=1}^{100} \operatorname{Edge}(t), \\
Q_{\text {max }} & =\max _{t=1 \ldots 100} \operatorname{Edge}(t) .
\end{aligned}
$$

Quantity $Q_{\text {fract }}$ is equal to the fraction of time horizons when fARIMA outperforms ARIMA, $Q_{\text {mean }}$ equals a mean advantage of fARIMA over ARIMA, while $Q_{\max }$ exposes the maximum edge of fARIMA algorithm over ARIMA. The larger the values of characteristics (5), the higher the advantage of using the fractional ARIMA model over the regular ARIMA for load prediction.

In order to inspect the influence of major scenario parameters on the Hurst exponent, we run a linear regression model using data from columns 2-5 of Table IV. The resulting equation is

$$
H=0.589-0.106 \gamma+0.00014 M+0.00017 N .
$$

The growth of number of stations $M$ and local fleet size $N$ increase the density and the total number of fast charging events. Therefore, the deseasonalized load becomes more selfsimilar with a higher Hurst exponent. At the same time, the growth of forgetfulness factor $\gamma$ decreases the total number of public charging events at public stations, making parameter $H$ lower.

Moreover, we compute correlation coefficients between $H$ and quality measures (5) based on columns 5-8 of Table IV:

$$
r\left(H, Q_{\text {fract }}\right)=0.90 ; r\left(H, Q_{\text {mean }}\right)=0.62 ; r\left(H, Q_{\text {max }}\right)=0.45
$$

Since all three coefficients are positive, then the advantage of using fARIMA algorithm expressed in terms of (5) grows when the Hurst exponent of the deseasonalized load $Y$ increases. This positive trend can be seen on Fig. 8-9 for all three quality indicators.

The conducted computational experiments illustrate the following patterns for simulated load time series:
Table IV

DESCRIPTION AND RESULTS OF EXPERIMENTS

\begin{tabular}{|l|c|c|c|c|c|c|c|}
\hline Exp & $\gamma$ & $\mathrm{M}$ & $\mathrm{N}$ & Hurst & $Q_{\text {fract }}$ & $Q_{\text {mean }}$ & $Q_{\max }$ \\
\hline \hline 1 & 0.05 & 50 & 100 & 0.629 & $66 \%$ & $2.33 \%$ & $5.76 \%$ \\
\hline 2 & 0.05 & 50 & 200 & 0.599 & $14 \%$ & $1.03 \%$ & $3.02 \%$ \\
\hline 3 & 0.05 & 50 & 300 & 0.652 & $100 \%$ & $2.81 \%$ & $6.81 \%$ \\
\hline 4 & 0.05 & 100 & 100 & 0.603 & $34 \%$ & $1.61 \%$ & $2.98 \%$ \\
\hline 5 & 0.05 & 100 & 200 & 0.642 & $100 \%$ & $1.96 \%$ & $5.69 \%$ \\
\hline 6 & 0.05 & 100 & 300 & 0.652 & $100 \%$ & $2.13 \%$ & $7.13 \%$ \\
\hline \hline 7 & 0.1 & 50 & 100 & 0.631 & $100 \%$ & $2.69 \%$ & $5.61 \%$ \\
\hline 8 & 0.1 & 50 & 200 & 0.615 & $63 \%$ & $2.31 \%$ & $6.43 \%$ \\
\hline 9 & 0.1 & 50 & 300 & 0.626 & $92 \%$ & $1.48 \%$ & $2.49 \%$ \\
\hline 10 & 0.1 & 100 & 100 & 0.573 & $10 \%$ & $1.85 \%$ & $5.39 \%$ \\
\hline 11 & 0.1 & 100 & 200 & 0.639 & $100 \%$ & $2.84 \%$ & $5.53 \%$ \\
\hline 12 & 0.1 & 100 & 300 & 0.661 & $100 \%$ & $2.25 \%$ & $2.81 \%$ \\
\hline \hline 13 & 0.2 & 50 & 100 & 0.596 & $7 \%$ & $0.11 \%$ & $1.18 \%$ \\
\hline 14 & 0.2 & 50 & 200 & 0.607 & $23 \%$ & $1.32 \%$ & $3.81 \%$ \\
\hline 15 & 0.2 & 50 & 300 & 0.614 & $72 \%$ & $1.10 \%$ & $3.49 \%$ \\
\hline 16 & 0.2 & 100 & 100 & 0.603 & $20 \%$ & $0.92 \%$ & $2.63 \%$ \\
\hline 17 & 0.2 & 100 & 200 & 0.625 & $88 \%$ & $2.14 \%$ & $5.13 \%$ \\
\hline 18 & 0.2 & 100 & 300 & 0.636 & $94 \%$ & $1.58 \%$ & $6.44 \%$ \\
\hline
\end{tabular}

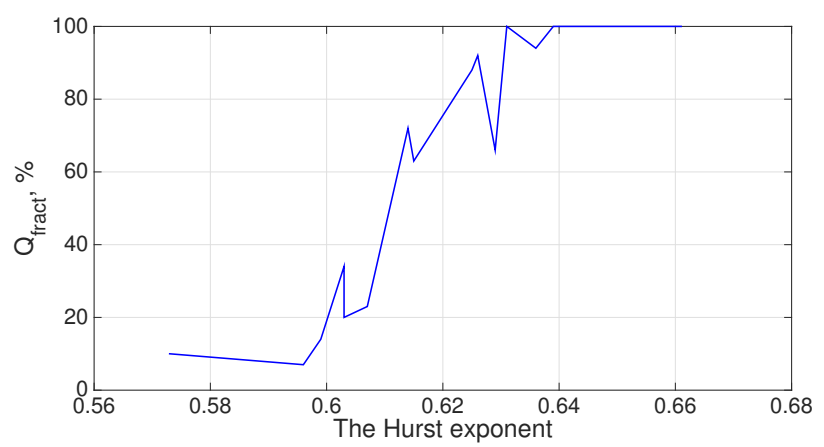

Figure 8. Advantage of fARIMA model in terms of $Q_{\text {fract }}$

- Deseasonalized loads $Y$ are self-similar processes with Hurst parameters $H$ strictly greater than 0.5 . Actually, $H \geq 0.573$ for considered scenarios.

- The fraction of horizons where the fitted fARIMA is more accurate than fitted ARIMA almost monotonically and rapidly grows as a function of $H$ (Fig. 8). For the Hurst parameters high enough $(H \geq 0.639)$ the indicator $Q_{\text {fract }}$ is equal to $100 \%$.

- Average fARIMA advantage $Q_{\text {mean }}$ is positive for all studied scenarios. For $H$ high enough $(H \geq 0.614)$ the mean fARIMA edge $Q_{\text {mean }}$ is securely greater than $1 \%$ what constitutes a significant forecasting edge (Fig. 9).

- Maximum advantage of fARIMA models $Q_{\max }$ varies from $1.18 \%$ to notable $7.13 \%$ for different multiplexing levels and time horizons $\tau$ (Fig. 9).

These observations allow us to conclude that self-similarity of the deseasonalized load $Y(t)$ plays an important role in load prediction problem. The higher the Hurst exponent $H$ is, the bigger a range of short-term forecasting time horizons where the fractional ARIMA model yields more accurate results than fitted ARIMA models. Probably, the main reason is that 


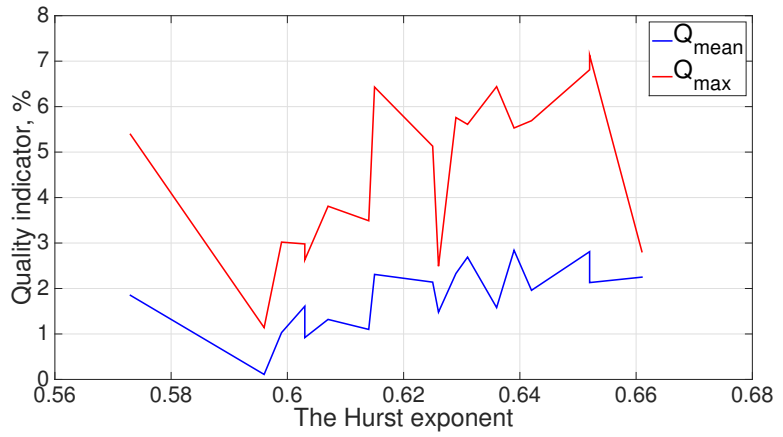

Figure 9. Advantage of fARIMA model in terms of $Q_{\text {mean }}$ and $Q_{\max }$

fARIMA models by definition take into consideration the selfsimilarity of the process $Y(t)$ and long-term dependencies between its consecutive values.

Some possible extensions of this work include implementation of probabilistic EV load forecasts instead of point predictions according to [23], incorporation of possibility to perform vehicle-to-grid power delivery [24] and consideration of probabilistic renewable and deterministic power grid energy sources at the same time [6].

\section{CONCLUSION}

This work addresses the problem of load modeling and prediction due to EV fast charging. Each charging station may generate electricity demand that can be as high as 120 $\mathrm{kW}$, which makes the aggregate load more irregular and difficult to be predicted. Therefore, forecasting of the load demand becomes a challenging and important problem for the electric utility because of its significant financial and safety consequences.

The paper starts with a discussion of empirical data characterizing EV owners driving and charging schedules. All necessary assumptions are explicitly stated and used in order to simulate activities of a fast charger. The output of the modeling algorithm is a time series that represents aggregate load of multiple fast charger stations.

The useful statistical properties of this load are discussed in the second part of the paper. The most important one is the self-similarity of the load demand after deseasonalizing and elimination of the linear trend. The Hurst exponent $H$ varies from 0.573 to 0.661 depending on the level of aggregation over a number of stations, a local fleet size and a forgetfulness factor value.

The main contribution of the paper is the empirical demonstration that the deseasonalized load is self-similar and that fARIMA model can produce more accurate short-term load forecasts. More precisely, the computational experiments support a statement that the higher the level of self-similarity of simulated load demand (described by the Hurst exponent $H$ ), the bigger the advantage of using fARIMA models. Having defined three quality characteristics of a forecasting algorithm, we show that all of them increase on average as a function of $H$. When $H$ is high enough $(H \geq 0.639)$ fitted fARIMA model outperforms all ARIMA algorithms in terms of RMSE for all forecasting time horizons up to 120 minutes. This edge is steadily greater than $1.1 \%$ and can be as high as $5-7 \%$ for some scenarios what constitues a significant advantage for electric utilities.

\section{REFERENCES}

[1] United Nations Quantified Economy-Wide Emissions Targets for 2020 [Online]. Available: http://unfccc.int/meetings/copenhagen_dec_ 2009/items/5264.php [June 12, 2015]

[2] K. Qian, C. Zhou et al., "Modeling of load demand due to EV battery charging in distribution systems," IEEE Trans. Power Systems, vol.26, no.2, pp.802-810, May 2011.

[3] T.-K. Lee, Z. Bareket et al., "Stochastic modeling for studies of realworld PHEV usage: driving schedule and daily temporal distributions," IEEE Trans. Veh. Technol., vol.61, no.4, pp.1493-1502, 2012.

[4] A. Rautiainen, S. Repo et al., "Statistical charging load modeling of PHEVs in electricity distribution networks using National Survey Data," IEEE Trans. Smart Grid, vol.3, no.4, pp.1650-1659, Dec 2012.

[5] Z. Duan, B. Gutierrez and L. Wang, "Forecasting Plug-In EV Sales and the Diurnal Recharging Load Curve," IEEE Trans. Smart Grid, vol.5, no.1, pp.527-535, Jan 2014.

[6] M. Simpson, T. Markel, "Plug-in electric vehicle fast charge station operational analysis with integrated renewables" (2012) [Online]. Available: http://www.nrel.gov/docs/fy12osti/53914.pdf [June 12, 2015]

[7] Idaho National Lab, "The EV project: Q3 2013 quarter report" [Online]. Available: http://avt.inl.gov/pdf/EVProj/EVProjInfrastructureQ32013.pdf [June 12, 2015]

[8] ECOtality, Idaho National Lab, "The EV project:'Q3 2011 report; The EV project," 11/7/11 [Online]. Available: http://avt.inl.gov/pdf/EVProj/ EVProjInfrastructureQ32011.pdf [June 12, 2015]

[9] Alternative Fuels Data Center [Online]. Available: http://www.afdc. energy.gov/data/widgets/10567 [June 12, 2015]

[10] Electric Drive Transportation Association, "EVs sales report" [Online]. Available: http://electricdrive.org/index.php?ht=d/sp/i/20952/pid/ 20952 [June 12, 2015]

[11] Alternative Fuels Data Center [Online]. Available: http://www.afdc. energy.gov/fuels/electricity_locations.html [June 12, 2015]

[12] R. Weron, "Modeling and forecasting electricity loads and prices: A statistical approach," John Wiley and Sons, 2006.

[13] W. A. Fuller, "Introduction to statistical time series," New York: John Wiley and Sons, 1976.

[14] D. Kwiatkowski, P. C. B. Phillips et al., "Testing the null hypothesis of stationarity against the alternative of a unit root," J. Econometrics, vol.54, pp.159-172, 1992.

[15] M. E. Crovella, A. Bestavros, "Self-similarity in world wide web traffic: evidence and possible causes," IEEE/ACM Trans. Netw., vol.5, pp.835846, Dec 1997.

[16] B. Mandelbrot, J. Wallis, "Noah, Joseph and operational hydrology," Water Resorces Res., vol.4, pp.909-918, 1968.

[17] J. Beran, "Statistics for long-memory processes. Monographs on statistics and applied probability," Chapman and Hall, New York, 1994.

[18] K. Park, "Self-similar network traffic and performance evaluation," John Wiley and Sons, New York, 2000.

[19] G. Box, G. Jenkins, G. Reinsel, "Time series analysis: Forecasting and control," John Wiley and Sons, Hoboken, 2007.

[20] R. J. Hyndman, G. Athanasopoulos et al., "Forecasting functions for time series and linear models", package 'forecast', [Online]. Available: cran.r-project.org/web/packages/forecast/forecast.pdf [June 12, 2015]

[21] J. Hosking, "Fractional differencing," Biometrika, vol.68, no.1, pp.165176, 1981.

[22] Y. Shu, Z. Jin et al. "Traffic prediction using fARIMA models," Proc. IEEE ICC'99, vol.2, pp.891-895, June 1999.

[23] T. Hong, J. Wilson and J. Xie, "Long Term Probabilistic Load Forecasting and Normalization with Hourly Information," IEEE Trans. Smart Grid, vol.5, no.1, pp.456-462, 2014.

[24] S. Inage, "Modelling Load Shifting Using EV in a Smart Grid Environment," International Energy Agency, 2010, [Online]. Available: https://www.iea.org/publications/freepublications/publication/load_ shifting.pdf [June 12, 2015] 


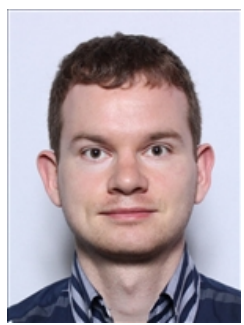

Nikita Korolko received his B.S. and M.S. degree in Mathematics from Novosibirsk State University, Novosibirsk, Russia. He is a $\mathrm{PhD}$ student at the Operations Research Center at Massachusetts Institute of Technology, Cambridge, MA, USA. His current research interests include robust modeling and optimization, adaptive optimization and their applications to online problems. He held research intern positions at Mitsubishi Electric Research Laboratories and Tesla Motors in 2014 and 2015, respectively.

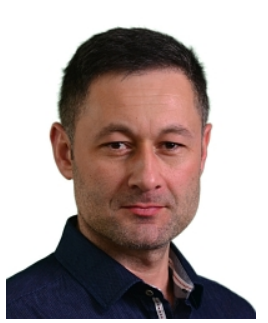

Zafer Sahinoglu received his MSc degree in 2001 and $\mathrm{PhD}$ in 2004 from New Jersey Institute of Technology in Biomedical Eng and Electrical Eng, respectively, and MBA from Massachusetts Institute of Technology Sloan School of Management in 2013. He worked at AT\&T's Shannon Research Labs as a visiting researcher in 2001, and then joined Mitsubishi Electric Research Labs. He has contributed and served in officer positions in numerous international standards in the areas of smartgrid, electric vehicles, indoor localization, wireless communications and sensor networks; and written two books published by Cambridge University Press. His current work includes remote sensing, robust modeling and optimization, battery modeling and energy big-data mining, and also development and integration of novel service businesses in productcentric companies.

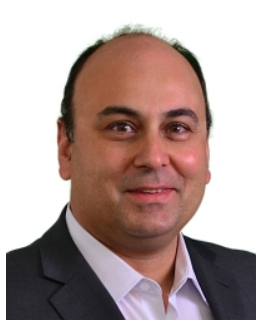

Daniel N. Nikovski received a Ph.D. degree in Robotics from Carnegie Mellon University in Pittsburgh, USA, in 2002. He is currently working at Mitsubishi Electric Research Laboratories in Cambridge, Massachusetts, USA. His research interests include machine learning, optimization and control, and numerical methods for analysis of complex industrial systems. 